\title{
FLOW-THROUGH MICRO-ELECTROPORATION CHIP FOR GENETIC ENGINEERING OF INDIVIDUAL CELLS
}

\author{
Yong Huang ${ }^{a}$ and Boris Rubinsky ${ }^{\mathrm{b}}$ \\ ${ }^{a}$ Bioelectronic Microsystems, Albany, CA94706 \\ ${ }^{b}$ Department of Mechanical Engineering, University of California, Berkeley CA 94720
}

\begin{abstract}
We describe a chip that is capable of performing gene transfer in individual biological cells through single cell microelectroporation. Microfluidic channels are used to handle individual cells in a flow through fashion that yields high electroporation efficiency. Controlled introduction of macromolecules and genetic engineering of an individual cell are demonstrated.
\end{abstract}

\section{INTRODUCTION}

There are many situations, in biology and biotechnology, in which it is desirable to transport across the cell membrane extracellular molecules that are normally cell membrane impermeant. For instance in genetic engineering, gene constructs, that are normally membrane-impermeant, need to be introduced into cells. Conventional methods for gene transfer through the cell membrane include the use of viral vectors, electroporation, liposome fusion and microinjection. These methods are applied to large populations of cells and do not have the capability to selectively manipulate particular cells of interest. Methods that operate on populations of cells are limited in their efficiency because there is no real time control over the events in each individual cell and therefore no real time feedback over the procedure [1][2].

We have developed a micro-electroporation chip [3][4], in which a live cell becomes an integral part of the chip's electrical circuit. The chip facilitates precise control over the process of electroporation in individual cells by measuring in real-time the electrical currents that flow through the cell membrane. This paper expands on our previous work by introducing a new chip design that uses microfluidic structures to precisely handle cells in a flowthrough fashion The chip performs electroporation facilitated gene transfer in each cell consecutively. In the chip, cells are transported through a micro channel, whose width is on the same order as the cells, in a single file to the vicinity of a micro hole. The cell near the hole is captured by pressure difference, incorporated in the electrical circuit of the chip, electroporated under controlled conditions, uploaded with the desired genetic material and than released to be replaced by the next cell, consequently yielding virtually $100 \%$ gene transfer rate in all the loaded cells.

Using the chip, we introduced a membrane impermeant nucleic acid stain, YOYO-1, into individual prostate adenocarcinoma (ND-1) cells. We performed a quantitative study on the electroporative delivery of macromolecule dyes under different electroporation conditions with fluorescent intensity analysis. Our results suggest that adjusting easily accessible electroporation parameters could regulate the amount of substances introduced into an individual cell. Micro-electroporation was also used to successfully transfect individual ND-1 cells with EGFP (Enhanced Green Fluorescent Protein) genes, and thereby demonstrates the feasibility of single cell level genetic manipulation and engineering.

\section{MICRO-ELECTROPORATION PRINCIPLES}

Electroporation is widely used in biotechnology for gene transfer. It employs transient high electrical field to temporarily permeabilize cell membranes by means of forming nanoscale pores in the lipid bilayer membrane[5][6]. As a consequence, DNA and other macromolecules, which are normally membrane-impermeant, can be transferred across the electroporated cell membranes [6][7][8]. Traditional electroporation technique uses two electrodes to apply high electrical field to a large number of cells in between. This is a trial and error procedure with no real time feedback and control over the electroporation process in individual cells. Therefore, a successful application heavily relies on empirical practice [7] and general effectiveness is very low with large variations.

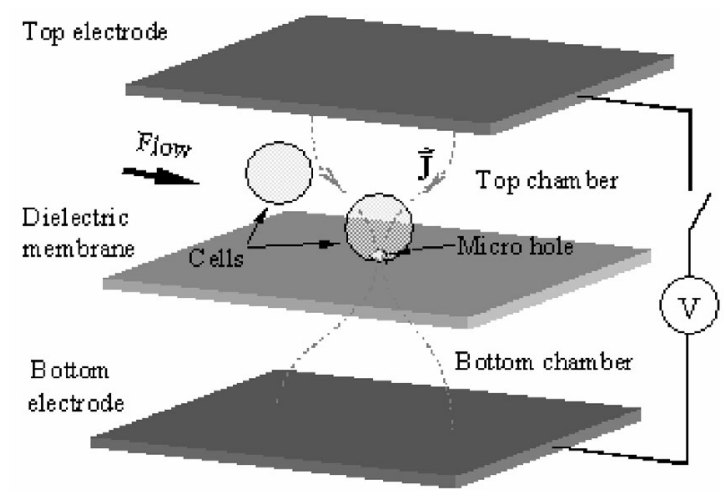

Figure 1. Schematic representation of single-cell level microelectroporation

It occurred to us that if an electroporated membrane becomes permeable to macromolecules, the pores in the membrane, which allow the transport of macromolecules, should also provide pathways for smaller ions to pass through the membrane; thereby generating measurable transmembrane currents during electroporation as a direct measure on membrane permeability. To measure the small transmembrane current, we came up with a design, shown in Figure 1, in which the electrical current between two electrodes is forced to pass through a micro hole in a dielectric membrane. When a cell is captured in the micro hole by an appropriate pressure gradient, the cell effectively blocks the current path between the two electrodes. Ideally, the transmembrane current is identical with the current flowing between two electrodes, which can be easily measured. Using the transmembrane current as a direct feedback on membrane permeabilization, the electroporation process of the captured cell can be precisely regulated. This controlled single-cell electroporation mode is referred as 'micro-electroporation.' A detailed description of the concepts and procedures of microelectroporation can be found in references [3][4]. 


\section{FLOW-THROUGH CHIP DESIGN AND FABRICATION}

Biotechnological applications require the manipulation of large numbers of cells. To this end we have developed a new micro-electroporation chip that can process large numbers of individual cells consecutively in a flow-through fashion. In the chip all cells are transported to the electroporation site in a single line profile through a micro channel whose width is slightly bigger than that of a cell. Once a cell is brought to the vicinity of the micro hole, it is captured by a suction pressure, then microelectroporated under controlled conditions, uploaded with desired foreign substances such as DNA, and finally released to be replaced by the next cell in the line. Because the microelectroporation method can achieve $100 \%$ electroporation effectiveness on each individual cells, virtually all cells loaded into the chip can be uploaded with the desirable gene constructs. Since the entire process can potentially be controlled by a computer, it is feasible to develop automated micro-electroporation systems for single-cell level genetic manipulation and drug delivery, with high efficiency and throughput.

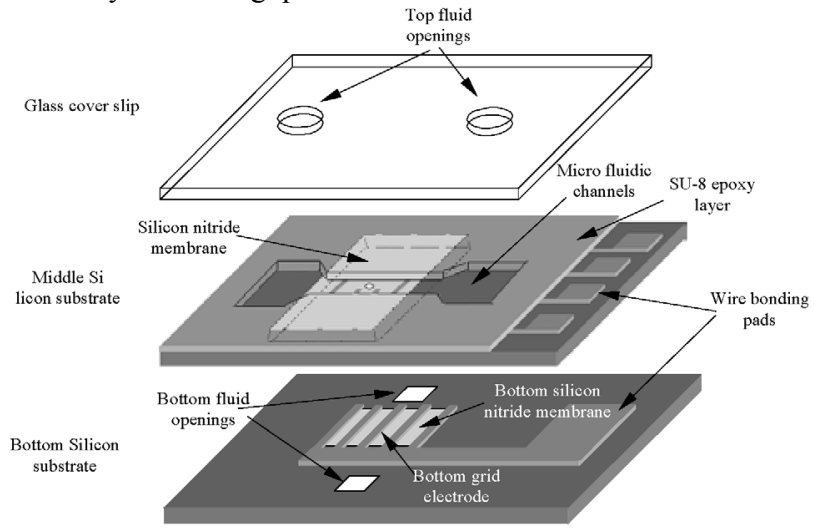

Figure 2. Construction of a flow-through micro-electroporation chip with microfluidic channels for precise cell transport

Figure 2 illustrates the construction of the flow-through micro-electroporation chip. The chip consists of two microfabricated silicon substrates and a glass cover slip, bonded together. The bottom and middle substrates form a bottom electroporation chamber that is filled with biological solutions through the bottom fluidic openings. The top electroporation chamber, where cells are loaded, is enclosed by the silicon nitride dielectric membrane and microfluidic structures on the middle substrate, and the cover slip. The central region of the chip is designed to be transparent so as to facilitate the use of light microscopy for visual observation of the cell manipulation process.

The middle silicon device is typically $20 \mathrm{~mm} \times 10 \mathrm{~mm} \times 500 \mu \mathrm{m}$ in size. It contains a $1 \mathrm{~mm} \times 1 \mathrm{~mm} \times 1 \mu \mathrm{m}$ silicon nitride dielectric membrane with one micro hole, sized between $2 \mu \mathrm{m}$ to $6 \mu \mathrm{m}$. The hole is etched through the nitride membrane with reactive ion etch. The micro hole provides the only fluidic and electrical connection between the fluid chambers and the electroporation electrodes. The microfluidic channel structures are made of SU-8 thick photoepoxy [9]. Figure 3 shows an optical image of a typical design of the flow-through micro-electroporation chip. The fluid channels are typically $30 \mu \mathrm{m}$ deep, $500 \mu \mathrm{m}$ wide at entries and converge to a $30 \mu \mathrm{m}$ wide, $200 \mu \mathrm{m}$ long micro channel near the micro hole region.

The micro channel, whose width is set to be 1.5 times the average diameter of cells used in our experiments, mechanically confines the loaded cells to pass by the micro hole, which is on the channel's centerline, one by one in a single line profile. Filtration structures (not shown) are placed near the entrances of the $30 \mu \mathrm{m}$ wide micro channel to prevent large particles and debris from entering and blocking the channel. At the entrance of the micro channel, there are two auxiliary electrodes that are used to further keep the channel from being blocked by large cells by degenerating the cells with high voltage pulses. Two more $10 \mu \mathrm{m}$ wide auxiliary microelectrodes are placed $10 \mu \mathrm{m}$ away on both sides of the micro hole for cell detection by impedance measurement.

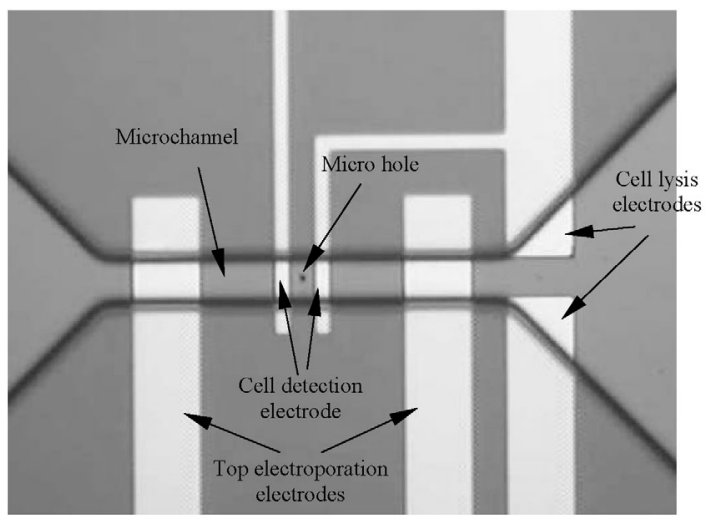

Figure 3.Typical layout of micro hole, microchannel and integrated electrodes on middle layer device
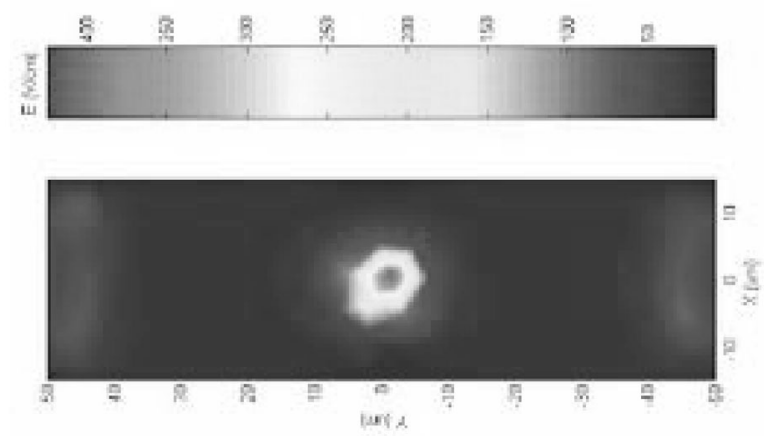

Figure 4. Electrical field inside a $30 \mu m \times 30 \mu m$ micro channel.

The illustrated plane is $5 \mu \mathrm{m}$ above the middle dielectric membrane. The micro hole is $3 \mu \mathrm{m}$ in diameter and locates at the channel center. Thickness of the middle dielectric membrane is $1 \mu \mathrm{m}$. Two 50 $\mathrm{mm}$ wide thin film electrodes are placed $50 \mu \mathrm{m}$ away from the micro hole. Distance between dielectric membrane and bottom electrode is $500 \mu \mathrm{m}$. Unit voltage is applied between top and bottom electrodes.

The top plate electrode in Figure 1 is substituted in our new design by two $50 \mu \mathrm{m}$ wide thin film platinum electrodes that are placed $100 \mu \mathrm{m}$ apart on two sides of the micro hole, as shown in Figure 3. This maintains a symmetric electrical field across the micro-hole. Simulation shows that the position of top electrodes has little influence on the electrical field distribution because most potential drop occurs in the vicinity of the micro hole. Figure 4 shows the simulated electrical field inside the flow-through chip. It is evident that the electrical field inside the channel is highly focused, with strong electrical field in the vicinity of the hole and a negligible field in other regions. This highly concentrated electrical field grants the chip great advantage in performing selective 
electroporation only on the cell captured in the micro hole while the nearby cells remain unaffected.

Final sealing of the microfluidic channel is achieved by bonding SU-8 structures to a glass cover with a UV-curable adhesive (NEA121, Norland products). The bonding process we developed produces excellent channel definition, sealing and bonding strength for channels down to $10 \mu \mathrm{m}$ wide. Figure 5 shows the bonding interfaces and a cross-section of a $50 \mu \mathrm{m} \times 20 \mu \mathrm{m}$ rectangular micro channel.

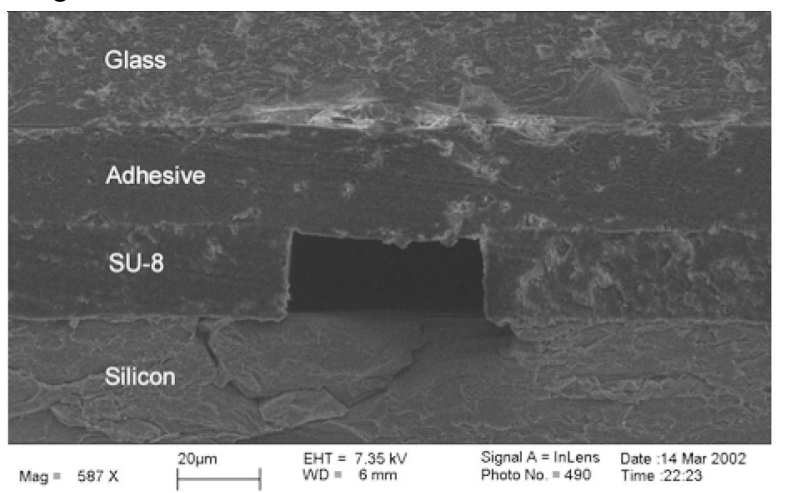

Figure 5. SEM cross-section image of a sealed $50 \mu \mathrm{m} \times 20 \mu \mathrm{m}$ rectangular micro channel and bonding interfaces

The bottom silicon layer, typically $23 \mathrm{~mm} \times 10 \mathrm{~mm} \times 500 \mu \mathrm{m}$ in size, hosts a bottom electrode, fluid openings and wire bonding pads. The bottom electrode is made of an array of thin film platinum lines, with typical width of $20 \mu \mathrm{m}$, line spacing of $50 \mu \mathrm{m}$, sitting on top of a $1 \mu \mathrm{m}$ thick transparent silicon nitride supporting membrane. Numerical simulation shows that the electrode geometry induced distortion in electrical field is localized within tens of micrometers from the electrode plane and beyond that, the geometry effects can be ignored and the grid electrode behaves as a plate electrode. The bottom layer is glued to the middle layer with a heat-curable adhesive (NEA121, Norland products). Finally, fluidic ports and tubing are attached to the assembled chip for fluid access.

\section{EXPERIMENT MATERIALS AND METHODS}

Human prostate adenocarcinoma cells (ND-1 cell line) are used in our experiments. For each experiment, cells from the confluent monolayer were removed by trypsinisation (trypsin $0.05 \%$, EDTA 0.025\%) (Gibco Grand Island, NY) and resuspended in Dulbecco's PBS (Sigma chemicals, St. Louis, MO) for experimental use.

In order to study the electroporation facilitated transfer of macromolecules, we used the YOYO-1 nucleic acid stain (Molecular Probes, OR). This macromolecule dye cannot pass normal cell membranes. However, when cells are electroporated, the dye molecules can enter the cells and make them fluorescent under excitation. The fluorescent intensity is a measure of how many dye molecules have entered the cells, therefore, the extent of dye transfer can be quantified by fluorescent intensity analysis. Typical YOYO-1 concentration used in our experiments was $1 \mu \mathrm{l} / \mathrm{ml}$. The excitation and emission spectra of YOYO-1 are $491 \mathrm{~nm}$ and $509 \mathrm{~nm}$ respectively.

In a typical experiment, ND-1 cell suspension with YOYO-1 dye is injected in the top microfluidic channels of a chip with a syringe. Visually assisted by a microscope (BX60, Olympus), a cell is pulled toward the micro hole along the micro channel, and then captured in the hole by applying a negative pressure in the bottom chamber. Then, an electrical pulse, which induces reversible electroporation in the cell, is applied [3][4]. After each pulse, the cells were exposed to UV light to generate a fluorescent image. Images of the fluorescing cell were recorded with a camera (Nikon) on Ektachrome transparency films (Kodak EPN100 Professional), which can accurately record color and intensity information. Subsequently the images were digitalized using a high accuracy transparency file scanner (Cannon FS2710) under the same scanning conditions. Intensity analysis was conducted on the fluorescent images to obtain the fluorescence intensity data for each individual cell. The data was used as the measure of the amount of uploaded fluorescent molecules under different control conditions.

\section{RESULTS AND DISCUSSIONS}

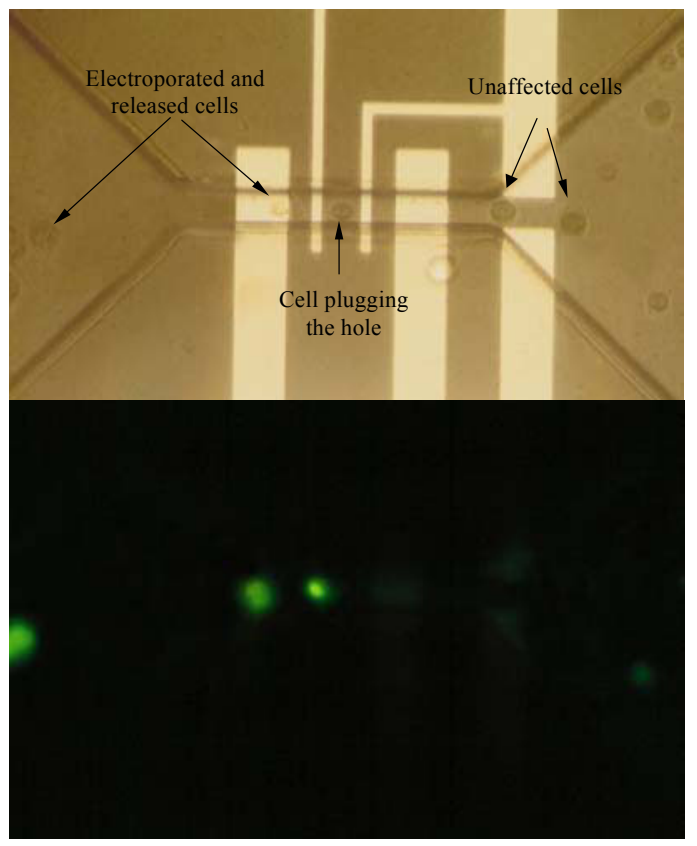

(a)

(b)

Figure 6. a) Bright field and b)fluorescent images of cells transported to the electroporation site along the micro channel in a single line profile

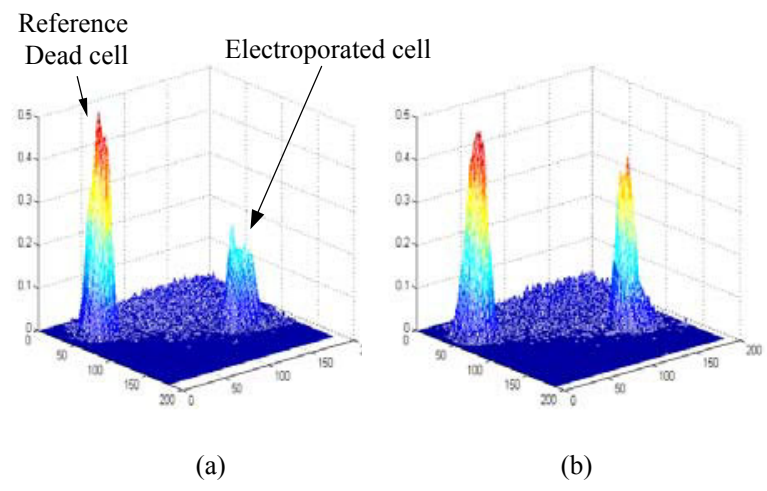

Figure 7. Fluorescence intensity plots for quantitative analysis of YOYO-1 macromolecule transfer a) after three 10V/100ms electroporation pulses b)after six 10V/100ms electroporation pulses

Controlled membrane transfer: Experiments have been carried out to study electroporation facilitated macromolecule 
transfer across individual cell membranes using the flow-through micro-electroporation chip.

Figure 6 shows typical images obtained from a chip with $3 \mu \mathrm{m}$ micro hole. Figure 6.a is a bright field image taken with a $10 \mathrm{X}$ objective that displays all cells in the view field. It can be seen that cells entered the $30 \mu \mathrm{m} \times 30 \mu \mathrm{m}$ micro channel from the left and march through the channel in a single file. A cell in the middle of the channel was trapped in the micro-hole where it was electroporated with a $10 \mathrm{~V} / 100 \mathrm{~ms}$ pulse. The cells to the left of the trapped cell are cells which have been released after being electroporated. It can be clearly seen in Figure 6.b, which is the corresponding fluorescent image, that only the trapped and released cells are fluorescent, indicating uptake of YOYO-1 dyes due to electroporation. The cells to the right which have not yet reached the electroporation site (micro hole) are not visible in the fluorescent image since the YOYO-1 dye has not been introduced inside those cells. This result demonstrates the chip's capability for highly selective single cell electroporation. This capability is due to the concentrated electrical field in the micro-hole vicinity as discussed above. In this particular image, the closest nonfluorescent cell is about $100 \mu \mathrm{m}$ away from the micro-hole electroporation site. However, in other experiments we found that a cell can remain non-fluorescent, i.e. not electroporated even when it is in contact with the cell trapped in the hole, which suggests a spatial selectivity resolution of about $10 \mu \mathrm{m}$.

Figure 7 demonstrates the quantitative analysis of dye transfer with fluorescent intensity analysis. Fluorescent images were obtained using the experimental procedure described above and than converted to intensity images. A dead cell that cannot exclude YOYO-1 dyes was used as a reference. The data from the dead cell is represented by the left plateaus in the intensity plots with average normalized values of 0.43 and 0.47 . Figure 7.a corresponds to the image taken after three $10 \mathrm{~V} / 100 \mathrm{~ms}$ electroporation pulse. The average fluorescent intensity of the target cell is 0.14 , with a standard variation of 0.02 , compared with no measurable intensity before electroporation pulses. This indicates that there is a considerable amount of YOYO-1 inside the cell. Figure $7 \mathrm{~b}$ shows that as more pulses were applied, at the sixth pulse the average fluorescence intensity of the cell increased to 0.22 , with the standard variation of 0.05 . This increase in intensity suggests that more YOYO-1 molecules have been introduced into the cells with each electroporation pulse. Because the relation between fluorescent intensity and dye concentration inside the cell is highly non-linear, more studies need to be done before

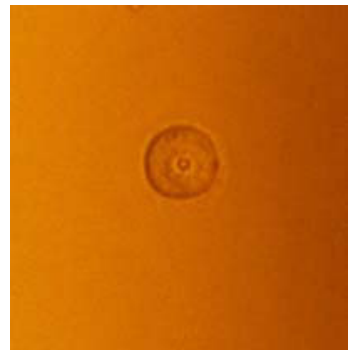

(a)

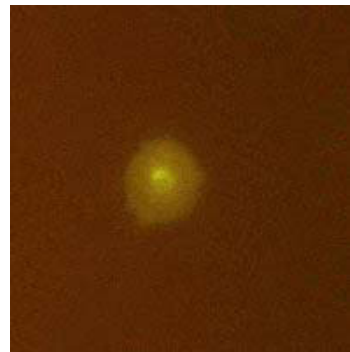

(b)
Figure 8. in situ gene transfection of individual ND-1 cell with micro-electroporation chip. A $3 \mu \mathrm{m}$ hole chip was used. Enhanced Green Fluorescent Protein (EGFP) gene was electroporatively introduced into the cell by applying two $10 \mathrm{~V} 100 \mathrm{~ms}$ square pulses, followed by 36 hours of in situ incubation of the cell in cell culture medium at $37^{\circ} \mathrm{C}$. a) bright field image before gene transfection b)fluorescent image of the EGFP transfected ND-1 cell quantitative interpretations of the intensity data can be made.

Single-cell genetic manipulation: The microelectroporation chip has the ability to introduce desirable macromolecules into specific individual cells. We have used the micro-electroporation chip, to perform a genetic manipulation on a single ND-1 cell inside the chip. The cell successfully expressed an EGFP gene which was inserted in the cell by microelectroporation. The detailed protocol is omitted here due to the limited space. This achievement demonstrates that with the microelectroporation chip, it is feasible to genetically engineer biological cells at the single cell level.

\section{CONCLUSIONS}

A new flow-through micro-electroporation chip has been described. The chip can be used for performing controlled electroporation in single cells in a flow-through fashion. Loaded cells are transported to the electroporation site by a microfluidic channel where they are and can be electroporated and permeabilized one by one. This results in a cell loading yield that is virtually $100 \%$ manipulation rate. Macromolecules, such as DNA, can be introduced into specific individual cells with the chip. The extent of membrane transfer can be regulated through readily adjustable electroporation parameters. Successful transfection of a ND-1 cell with EGFP gene is demonstrated to validate the feasibility of single-cell genetic manipulation.

\section{ACKNOWLEDGEMENTS}

This work is partially supported by NIH under Grant No. 1 R21 RR15252-01.

\section{REFERENCES}

1. I. Hapala, "Breaking the barrier: methods for reversible permealization of cellular membranes," Critical Reviews in Biotechnology 17, 105-122, 1997.

2. A. G. Sabelnikov, "Nucleic Acud Transfer Through Cell Membranes: Towards the Underlying Mechanisms." Prog. Biophys. Molec. Biol. 62: 119-152, 1994

3. Y. Huang and B. Rubinsky, "Micro-electroporation: improving the efficiency and understanding of electrical permeabilization of cells," Biomedical Microdevices 3, 145-150, 2000.

4. Y. Huang and B. Rubinsky, "Microfabricated electroporation chip for single cell membrane permeabilization." Sensors and Actuators: Physical, 89, 242-249, 2001.

5. J.C. Weaver, "Electroporation: A general phenomenon for manipulating cells and tissues, "Journal of Cellular Biochemistry 51, 426-435, 1993.

6. E. Newman, A.E Sowers, C. A. Jordan, Electroporation and electrofusion in cell biology, Plenum Press, New York, 1989.

7. G. L. Andreason, "Electroporation as a technique for the transfer of macromolecules into mammalian cell lines, "J. Tiss. Cult. Meth. 15, 56-62, 1993.

8. $\quad$ P.F. Lurquin, "Gene transfer by electroporation.", Molecular Biotechnology 7:5-35, 1997

9. H. Lorenz, M. Despont, N. Fahrni, J. Brugger, P. Vettiger, P. Renaud, "High-aspect-ratio, ultrathick, negative-tone near-UV photoresist and its applications for MEMS," Sensors and Actuators:Physical, 64, pp.33-39, 1998. 